\title{
Percutaneous pedicle screw fixation of the lumbar spine
}

\author{
Kevin T. Foley, M.D., SAnjay K. Gupta, M.D., JefF R. Justis, B.S., \\ and Michael C. Sherman, M.S. \\ Department of Neurosurgery, Image-Guided Surgery Research Center, University of Tennessee, \\ Memphis, Tennessee
}

\begin{abstract}
Object. Standard techniques for lumbar pedicle screw fixation involve open exposures and extensive muscle dissection. The purpose of this study was to report the initial clinical experience with a novel device for percutaneous posterior fixation of the lumbar spine.

Methods. An existing multiaxial lumbar pedicle screw system was modified so that screws could be placed percutaneously by using an extension sleeve that would allow for remote manipulation of the polyaxial screw heads and remote engagement of the screw locking mechanism. A unique rod insertion device was developed that linked to the screw extension sleeves, allowing for a precut, precontoured rod to be placed through a small stab wound. Because the insertion device relies on geometrical constraint of the rod pathway through the screw heads, rods can be placed in a standard submuscular position with minimal manipulation, essentially no muscle dissection, and without the need for direct visual feedback. Twelve patients (six men and six women who ranged in age from 23-68 years) underwent pedicle screw fixation in which the rod insertion device was used. Spondylolisthesis was present in 10 patients and nonunion of a prior interbody fusion was present in two. All patients underwent successful percutaneous fixation. Ten patients underwent single-level fusions (six at L5-S1, three at L4-5, and one at L2-3), and two underwent two-level fusions (one from L-3 to L-5 and the other from L-4 to S-1). The follow-up period ranged from 3 to 12 months (mean 6.8 months).

Conclusions. Although percutaneous lumbar pedicle screw placement has been described previously, longitudinal connector (rod or plate) insertion has been more problematic. The device used in this study allows for straightforward placement of lumbar pedicle screws and rods through percutaneous stab wounds. Paraspinous tissue trauma is minimized without compromising the quality of spinal fixation. Preliminary experience with this device has been promising.
\end{abstract}

\section{KEY WORDS - percutaneous surgery - lumbar spine • pedicle - screw fixation}

The use of pedicle screws for spinal stabilization has become increasingly popular worldwide. Pedicle screw systems engage all three columns of the spine and can resist motion in all planes. Several studies suggest that pedicle screw fixation is a safe and effective treatment for many spinal disorders. ${ }^{4,13}$ Standard techniques for pedicle screw placement, however, require extensive tissue dissection to expose entry points and to provide for lateral-tomedial orientation for optimal screw trajectory. Open pedicle fixation and spinal fusion have been associated with extensive blood loss, lengthy hospital stays, and significant cost. ${ }^{11}$

The purpose of this paper is to describe a technique and instrumentation designed by the senior author (K.T.F.) for minimally invasive posterior fixation of the lumbar spine by using percutaneous screws and rods (Sextant; Medtronic Sofamor Danek, Memphis, TN). Our initial clinical experience will also be included. Although percutaneous

Abbreviations used in this paper: $\mathrm{AP}=$ anteroposterior; $\mathrm{CT}=$ computerized tomography; MED = microendoscopic discectomy. lumbar pedicle screw insertion has been previously reported,,$^{5-7}$ a minimally invasive approach to inserting a longitudinal connector for these screws has proven more challenging. The Sextant system allows for the straightforward placement of lumbar pedicle screws and rods through percutaneous stab wounds. The screws and rods are placed in an anatomical position similar to that achieved by an analogous open surgical approach. Paraspinous tissue trauma is greatly minimized without sacrificing the quality of the spinal fixation. Our preliminary experience with this technique has been promising.

\section{CLINICAL MATERIAL AND METHODS}

\section{Patient Population}

Preoperatively, all patients presented with mechanical back pain. Radiculopathy, when present, was due to entrapment of an exiting nerve root within a collapsed neuroforamen. Appropriate conservative management had failed to relieve patients of their symptoms. There were six men and six women; ages ranged from 23 to 68 years. 


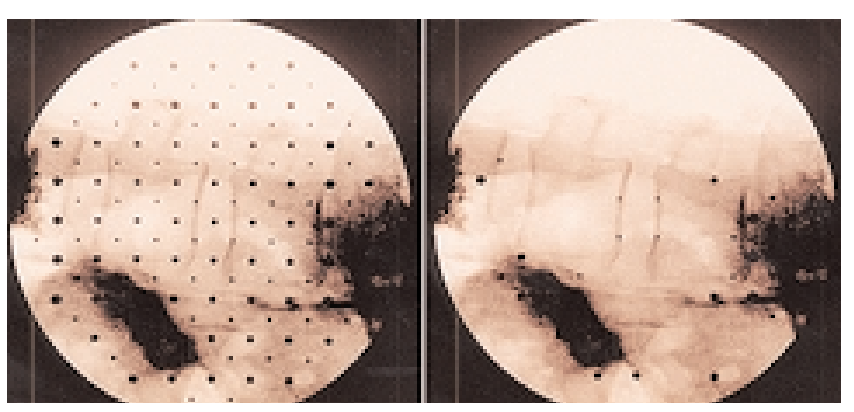

Fig. 1. Left: Lateral fluoroscopic image of the lumbar spine obtained using the FluoroNav system. Note the calibration balls superimposed on the image. Right: The image has been automatically calibrated using the FluoroNav software. The computer subtracts the calibration balls from the image during this process.

Radiographic indications were correlated with clinical indications. Grade I spondylolisthesis was present in six patients; Grade II, in three; and Grade III, in one. Two patients presented with nonunion after a previous fusion. Ten patients required a single-level fusion, and two patients required a two-level fusion. Of the single-level fusions, one was at L2 -3 , three were at $\mathrm{L} 4-5$, and six were at L5-S1. One of the two-level fusions was from L-3 to L-5 and the other was from L-4 to S-1.

\section{Patient Positioning and Operating Room Setup}

Percutaneous posterior fixation of the lumbar spine can be performed after induction of general or epidural anesthesia. Thereafter, the patient is positioned prone on top of chest rolls with the abdomen free. A C-arm fluoroscopy device is used for guidance of percutaneous screw placement. Although conventional fluoroscopy can be used for this purpose,,$^{5}$ it has certain disadvantages: the inability to visualize more than one plane of view at a single time (when using a single fluoroscope), the ergonomic challenges of working around a $\mathrm{C}$-arm, and radiation exposure. ${ }^{8-10}$ Instead, we have used virtual fluoroscopy (FluoroNav; Medtronic Surgical Navigation Technologies, Louisville, $\mathrm{CO}$ ) for guiding screw placement, because it avoids the disadvantages of conventional fluoroscopy (see Virtual Fluoroscopy). Regardless of whether one uses conventional or virtual fluoroscopic guidance for the procedure, it is important to check that adequate AP and lateral fluoroscopic images of the lumbar spine can be obtained before preparing and draping the patient.

\section{Virtual Fluoroscopy}

Virtual fluoroscopy combines an image-guided surgical computer and C-arm fluoroscopy. ${ }^{1,2,9} \mathrm{~A}$ light-emitting diode-fitted calibration target is applied to the C-arm. An optical camera is used to track the fluoroscope as well as a spinal dynamic reference array and various spine surgery tools including a pedicle awl, a pedicle probe, bone taps, and a screwdriver. Fluoroscopic images of the lumbar spine are obtained, sent to, and saved in the imageguidance computer and automatically calibrated (Fig. 1). The process is independent of surgical exposure and enables percutaneous spinal registration. The real-time location of the tracked tools is graphically projected onto the

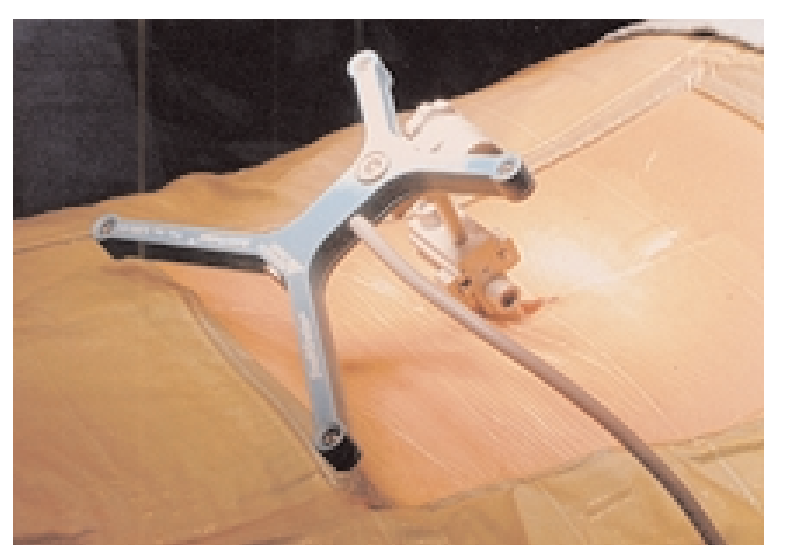

Fig. 2. Photograph. A dynamic reference array is rigidly attached to the patient. The reference array is fixed to the spinous process one level above the fusion site via a stab incision.

preacquired fluoroscopic images. These preacquired images can be obtained in multiple planes (that is, AP, lateral, and oblique), and the tools can be tracked simultaneously on all images. The advantages of virtual fluoroscopy over a conventional $\mathrm{C}$-arm unit include this simultaneous multiplanar guidance capability, decreased radiation exposure, ${ }^{8}$ and the ability to quantify radiographically obtained information. ${ }^{1,2}$

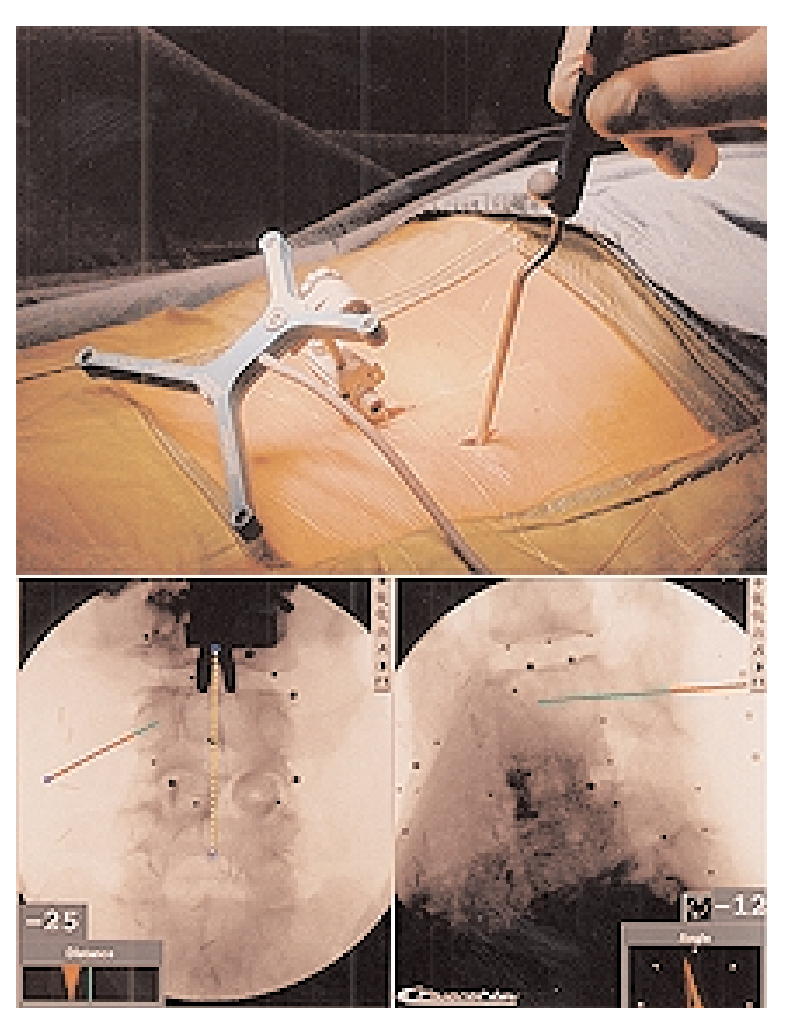

Fig. 3. Upper: Photograph showing the sharp tip probe being used to localize a pedicle trajectory. Lower: Virtual fluoroscopic images demonstrating the virtual probe (red line), representing the sharp tip probe itself, projected on previously acquired AP and lateral fluoroscopic images of the lumbar spine. The green line shows a virtual forward projection of the probe's trajectory into the pedicle. 


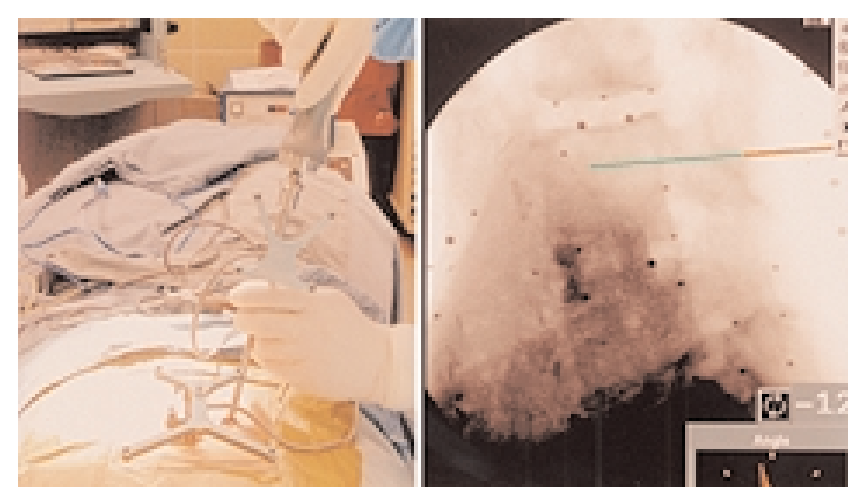

Fig. 4. Left: A light-emitting diode-equipped awl-probe-tap is used to create a pedicle pilot hole under virtual fluoroscopic guidance. Right: Virtual fluoroscopic image demonstrating that the probe (red line) can be virtually extended (green line) into the pedicle; the computer quantifies the length of this extended pathway. The appropriate length for a pedicle screw can thus be chosen prior to its insertion.

\section{Initial Incision and Pedicle Identification}

If virtual fluoroscopic guidance is used, a dynamic reference array is first inserted. A small incision is made over the spinous process at the level above the planned segmental fusion site. Lateral fluoroscopy is used to localize this spinous process, and the incision is brought down through the fascia. A percutaneous reference array is then attached to the spinous process (Fig. 2). Fluoroscopic images are obtained in the AP and lateral planes, making sure the pedicles can be adequately visualized. If necessary, oblique ("owl's-eye") views can be obtained as well. These images are automatically calibrated (activated) using the FluoroNav software.

With the FluoroNav sharp tip probe, entry points are chosen for the pedicles to be instrumented. The instrument tip can be virtually extended with the software so as to

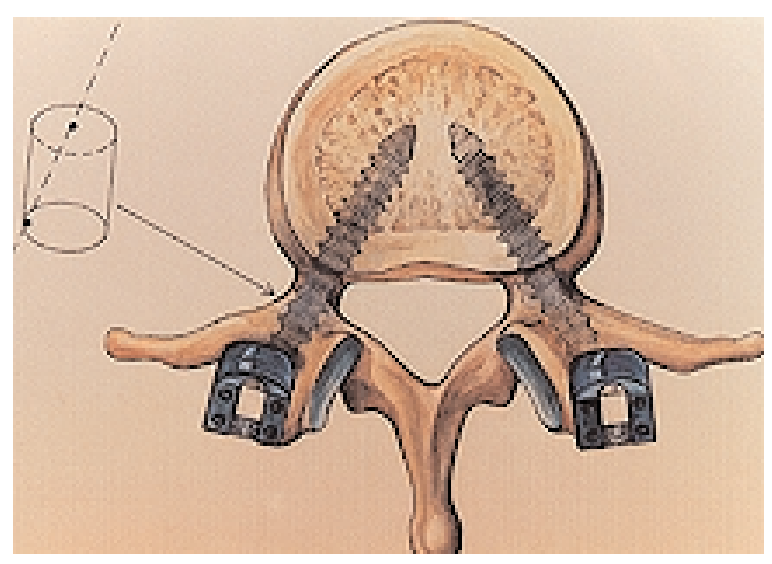

Fig. 5. Artist's drawing. A pedicle can be conceptualized as a cylinder. If a proposed screw trajectory enters the top of this cylinder (the pedicle screw entry site) at or medial to its lateral wall and exits the bottom of the cylinder (the junction of the pedicle and the vertebral body) lateral to its medial wall (enough to accommodate the anticipated screw diameter), the pedicle can be safely navigated. choose an ideal trajectory that traverses the underlying pedicle (Fig. 3). An approximately 15-mm incision is made at the skin entry point and extended into the underlying subcutaneous tissue. A K-wire is used to perforate the fascia and a series of sequential dilators are then used to dilate the fascia and to separate bluntly the underlying paraspinous muscles down to the spine. ${ }^{3}$ The dilators are removed, and a tracked awl and a tracked pedicle probe are used to create a pedicle pilot hole under virtual fluoroscopic guidance (Fig. 4). Using the FluoroNav software, both the length and diameter of the pedicle screw may be chosen at this time.

\section{Pedicle Screw Placement}

Under real-time guidance with the multiplanar virtual fluoroscopy images, the chosen pedicles are tapped and screws are placed. A thorough knowledge of pedicle anatomy and the sagittal and axial angulation of the individual pedicles is mandatory for safe percutaneous screw placement. ${ }^{12}$ These angles are best judged using preoperative CT or magnetic resonance imaging of the lumbar region. We find biplanar virtual fluoroscopic guidance of the transpedicular trajectory to be helpful. The pedicle is a roughly cylindrical structure. If one enters the pedicle at its lateral margin and exits the structure lateral to its medial wall (enough to accommodate the anticipated screw diameter), the pedicle can be navigated safely (Fig. 5). Such a trajectory can be chosen by virtually extending the tip of the pedicle awl or probe by using the FluoroNav software prior to traversing the pedicle. The tip is extend-

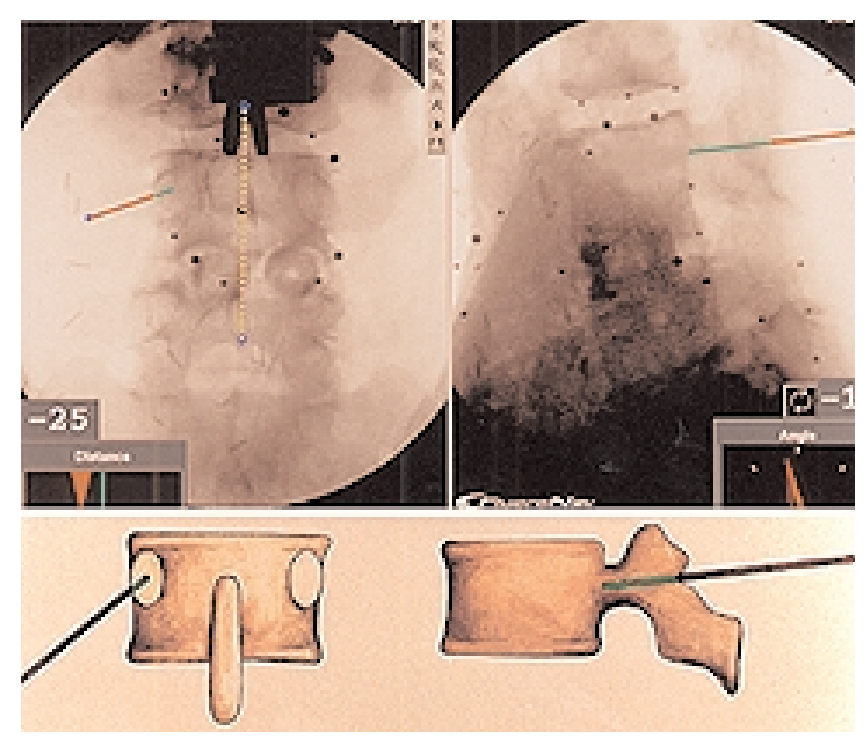

Fig. 6. Upper: The light-emitting diode-equipped awl-probetap is placed at the pedicle entry point. The virtual probe (red line) can be seen on the AP and lateral virtual fluoroscopic views. The probe's proposed trajectory is then extended (green line) to ensure that, as the probe reaches the base of the pedicle, it will lie safely within the pedicle cylinder on the AP and lateral views. Lower: Artist's drawing illustrating the aforementioned concept. The red line is the probe itself and the green line is the forward projected trajectory. The probe's position can be adjusted until the proposed trajectory is acceptable, after which the probe is advanced through the pedicle. 


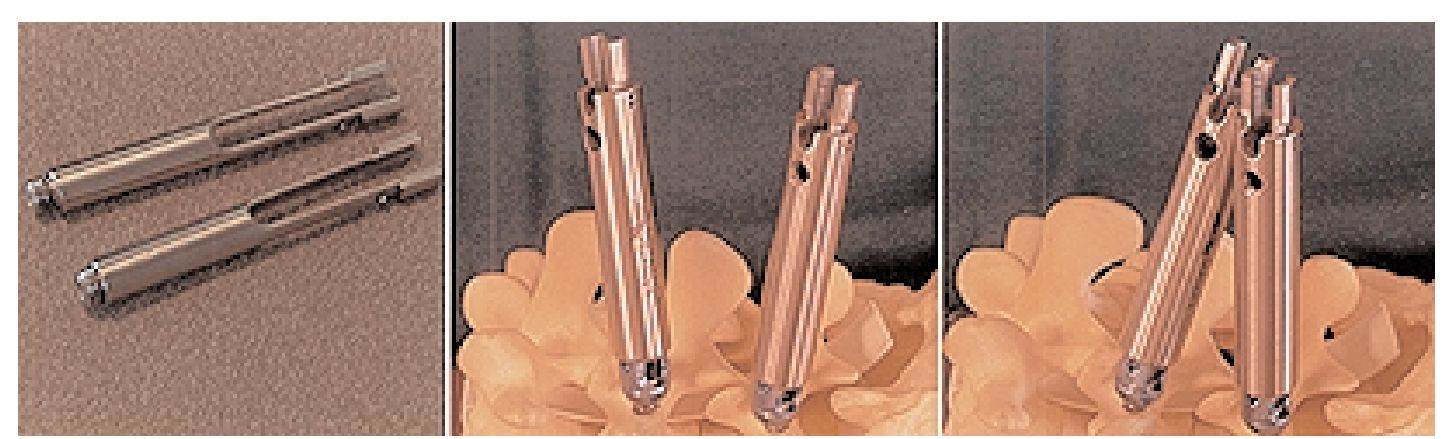

Fig. 7. Photographs. Left: The screw extender inner sleeve is preloaded with the lock plug. The lock plug will ultimately secure the rod to the screw. Center: A multiaxial screw head can be manipulated with the screw extenders even after the screw is inserted. Right: The screw heads are manipulated (angulated and rotated) and aligned to accommodate the trajectory of the Sextant rod. This alignment occurs automatically when the screw extenders are connected.

ed on the lateral fluoroscopic view until it reaches the pedicle-vertebral body junction, keeping the sagittal angulation of the instrument aligned so that its trajectory bifurcates the pedicle on the lateral view. The axial angulation of the instrument is adjusted until the extended tip is seen within the boundaries of the pedicle on the AP view but lateral to the medial pedicle wall (Fig. 6).

Alternatively, the pedicle can be navigated using a conventional C-arm fluoroscope that is alternated between AP, lateral, and oblique views. If this technique is chosen, one must obtain multiple sequential views of the pedicle probe in at least two planes as it is advanced down the pedicle. ${ }^{4,12}$ It is important to keep these trajectories in mind to ensure the accuracy of percutaneous screw placement. One advantage of percutaneous screw placement over the conventional open technique, however, is that it is much easier to achieve the required medial angulation,

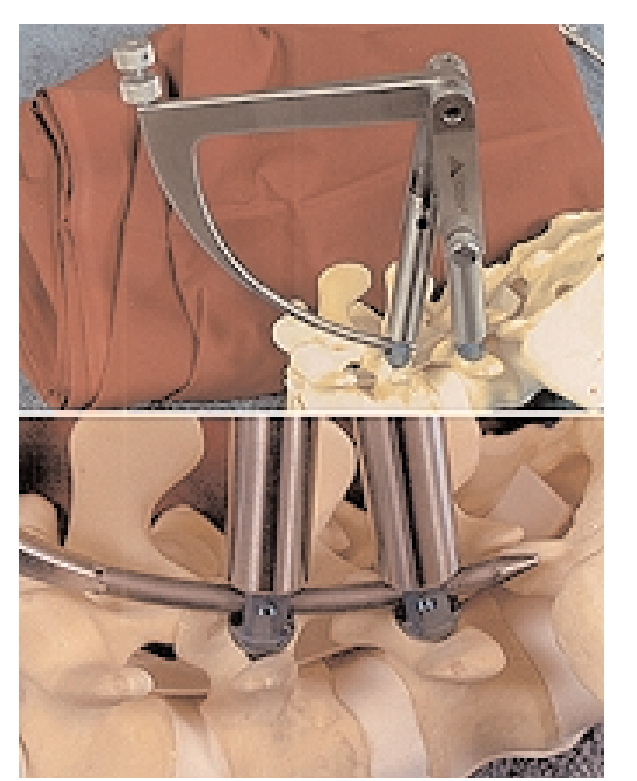

Fig. 8. Upper: The inserter-rod combination follows the curvilinear path connecting both screw head openings. Lower: The precontoured rod is seated in the screw heads in a reproducible, predictable fashion. because extensive soft-tissue and muscle retraction is avoided.

\section{Rod Placement}

The multiaxial Sextant pedicle screws are attached to screw extenders. These screw extenders have inner and outer sleeves. The inner extender sleeve is designed to be preloaded with a lock plug (Fig. 7 left). This lock plug will eventually connect the screw to the rod. The outer sleeve actually extends over the multiaxial screw head. The inner sleeve starts in a first position that allows the lock plug to be partially advanced into the multiaxial screw head, which connects the screw to the extender sleeve combination. The screw head remains mobile on its shank. Thus, the screw head can be manipulated remotely (rotated and angulated) by moving the far end of the screw extender even after the screw is placed within the pedicle (Fig. 7 center and right).

After a pair of pedicle screws, together with their attached extenders, has been inserted, a Sextant rod is placed. The Sextant rods are precontoured into a curvilinear shape that precisely matches the contour of the Sextant rod inserter. The rods are designed to fix rigidly to the inserter, forming a smooth arc. Additionally, the Sextant inserter attaches to the screw extenders. The resulting ar-

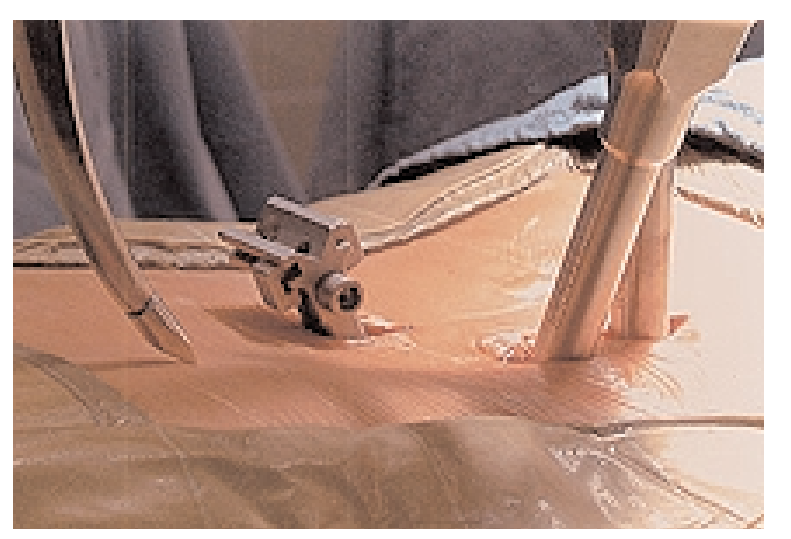

Fig. 9. A trochar tip is attached to the rod inserter to pierce the fascia prior to placement of the rod. A stab incision is made where the trochar intersects the skin. 


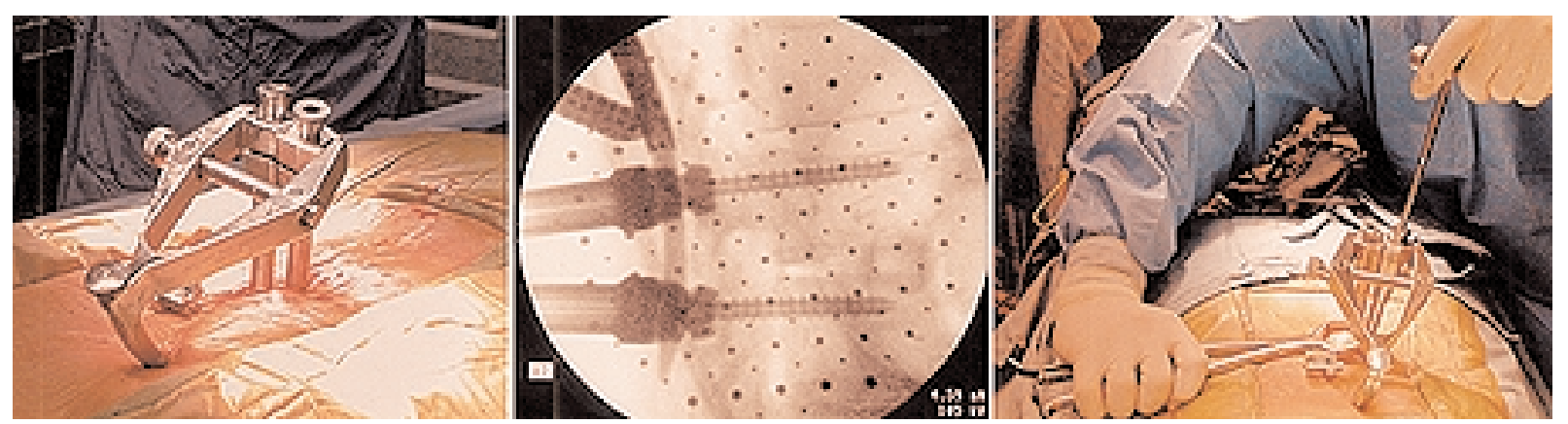

Fig. 10. Left: Photograph showing the rod inserter after the rod is in final position. Center: After placement of the rod, with the inserter still in place, a fluoroscopic image is obtained to confirm placement and proper rod length. Right: Prior to final tightening of the screw/rod combination, appropriate forces (in this case, compression) can be applied.

rangement resembles the navigational device of the same name.

The screw extenders are aligned at their proximal ends (Video 1). This maneuver arranges the distal ends, which are connected to the multiaxial screw heads, in a way that allows the openings in the screw heads to fit the same curvilinear path of the precontoured rod. The geometrical configuration is such that this path is identical to the arc created by the union of the rod and the Sextant rod inserter. In fact, once the joined screw extenders are attached to the rod inserter, this geometrical relationship is constrained. The arc, subtended by the inserter-rod combination, must now follow the path connecting both screw heads (Fig. 8).

Click here to view Video 1. By connecting the screw extenders at their proximal ends, the screw heads become positioned such that the openings in them are aligned to allow the placement of the precontoured Sextant rod.

After the screw extenders have been connected to the Sextant rod inserter, a trochar tip is attached to the insert-

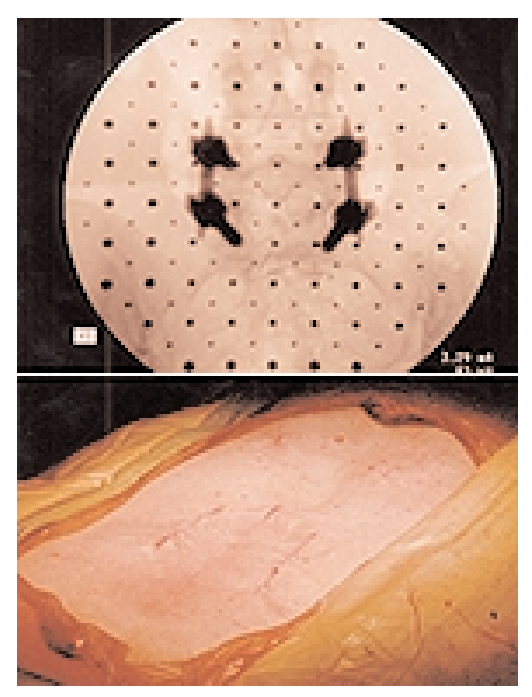

Fig. 11. Upper: A final AP fluoroscopic view obtained after percutaneous placement of pedicle screws and rods. Lower: The stab wounds are closed with a subcuticular suture. er (Fig. 9). The skin is marked where this tip intersects it, and a small stab wound is made with a No. 15 blade. The trochar tip serves to open the underlying fascia. Once the fascia has been penetrated, the tip is removed and a Sextant rod is attached. The rod is inserted through the same stab wound and intersects the screw heads (Video 2). This is checked fluoroscopically (Fig. 10 left and center). Appropriate forces (compression and/or distraction) can be applied to the construct prior to final tightening (Fig. 10 right). The inner sleeves are now advanced to their second position, allowing a hex driver to be inserted and tighten the lock plugs. The lock plugs are designed with a torquelimiting breakoff. This allows simultaneous locking of the rod to the screw while the extension sleeve detaches. The Sextant itself serves as a counter-torque device. The rod is remotely released from the Sextant inserter and the inserter is removed from the field, leaving a percutaneous rod/ screw combination in place (Fig. 11). The procedure can be repeated on the contralateral side of the spine, after which the stab incisions are irrigated and closed.

Video 2. Once the fascia is opened with the trochar tip, a Sextant rod of appropriate length is connected to the inserter. This rod passes through the same trajectory as the trochar and then intersects the screw heads along a geometrically constrained pathway.

\section{RESULTS}

The initial clinical results when using the Sextant system for percutaneous posterior fixation of the lumbar spine have been promising. This is a new procedure and

TABLE 1

Summary of preoperative findings and fusion levels

\begin{tabular}{lc}
\hline \multicolumn{1}{c}{ Characteristic } & No. of Patients \\
\hline male & 6 \\
female & 6 \\
preop findings & 6 \\
Grade I spondylolisthesis & 3 \\
Grade II spondylolisthesis & 1 \\
Grade III spondylolisthesis & 2 \\
nonunion of prior fusion & \\
no. of fusion levels & 10 \\
1 & 2 \\
2 &
\end{tabular}


TABLE 2

Modified Macnab criteria for characterizing outcome after spinal surgery

\begin{tabular}{|c|c|}
\hline Result & Criteria \\
\hline excellent & $\begin{array}{l}\text { no pain; no restriction of mobility; return to normal } \\
\text { work \& level of activity }\end{array}$ \\
\hline good & $\begin{array}{l}\text { occasional nonradicular pain; relief of presenting } \\
\text { symptoms; return to modified work }\end{array}$ \\
\hline fair & $\begin{array}{l}\text { some improved functional capacity; still handicapped } \\
\text { \& unemployed }\end{array}$ \\
\hline poor & $\begin{array}{l}\text { continued objective symptoms of root involvement; } \\
\text { additional op intervention needed at the index } \\
\text { level irrespective of length of postop follow up }\end{array}$ \\
\hline
\end{tabular}

the results are only preliminary. Twelve consecutive patients underwent placement of percutaneous pedicle screws and rods (Table 1). All of the percutaneous posterior fixation procedures were preceded by a fusion that was performed during the same operative session. In 10 patients an anterior lumbar interbody fusion was first performed, ${ }^{14}$ in one patient interbody fusion was performed via a minimally invasive lateral retroperitoneal approach, and in another the MED tubular retractor system was used for a percutaneous onlay fusion at L5-S1. ${ }^{3}$ Data were collected in a prospective manner.

One complication was noted. A lock plug loosened in one patient early in the clinical series that necessitated an outpatient surgery for replacement of the loosened hardware. This was an asymptomatic event, noted on routine follow-up radiographs; a solid fusion resulted, and the patient experienced a good clinical outcome. Based on this incident, the lock plugs were redesigned; no other incidents of loosening have been noted.

All operations were performed using the aforementioned technique. The operative time ranged from 90 to 220 minutes, with the longer operative times occurring early in the learning curve. Fifty percent of the patients were discharged on postoperative Day 1 or 2; the remaining patients were discharged on postoperative Day 3. Interestingly, the longer hospital stays typically related to ileus secondary to an anterior approach for fusion.

The follow-up period ranged from 3 to 12 months (mean 6.8 months). All patients improved clinically, and

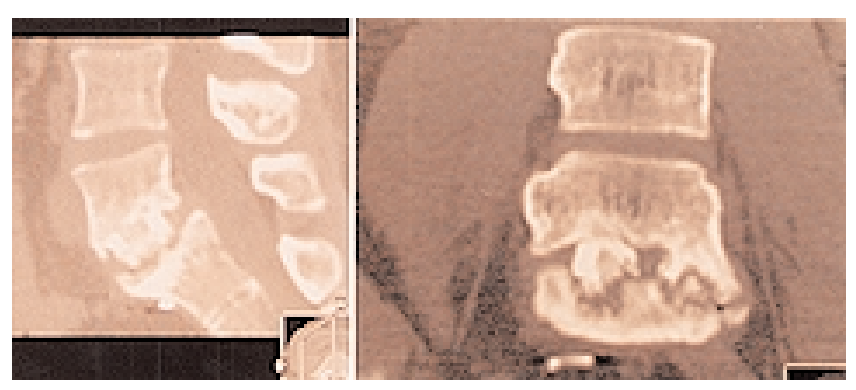

Fig. 12. Reconstructed CT studies. Left: Sagittal scan demonstrating nonunion at the graft-sacrum junction. The patient had undergone an L5-S1 laparoscopic interbody fusion 1 year prior. Right: Coronal scan also revealing nonunion.

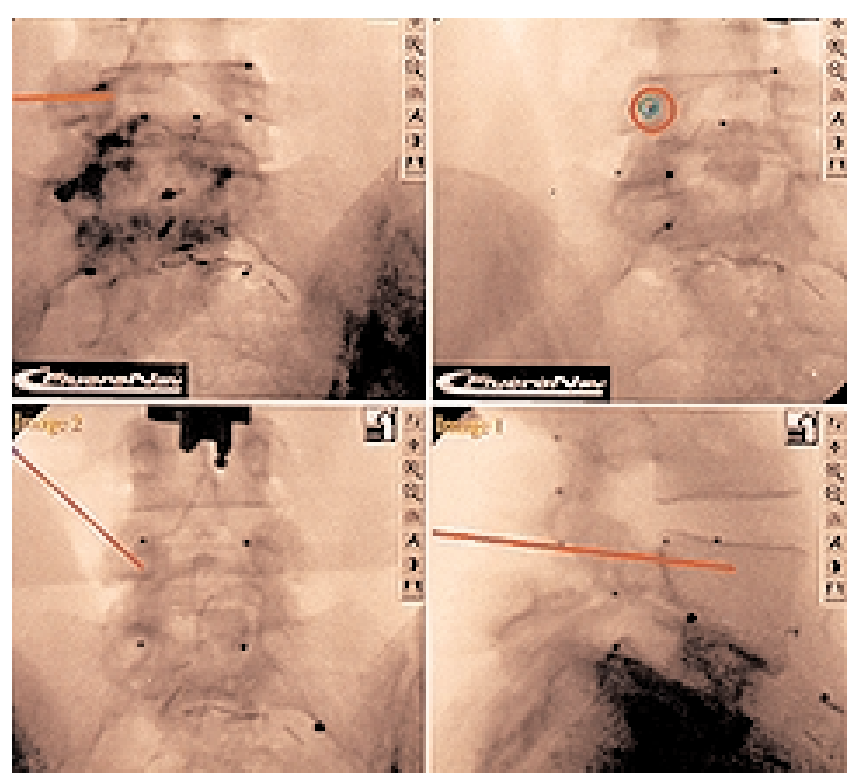

Fig. 13. Multiplanar virtual fluoroscopic images were used for placement of the percutaneous pedicle screws. Here, the trajectory for the left L-5 screw is demonstrated.

outcome was classified using the modified Macnab criteria (Table 2). Results were considered excellent in six patients, good in five patients, and poor in one patient. Although this latter patient fared well clinically, he required reoperation for hardware revision as previously noted. Of the seven patients who had been followed for longer than 6 months, all were judged to have solid fusions.

\section{ILLUSTRATIVE CASE}

This 23-year-old woman presented with recurrent mechanical low-back pain after having undergone a laparoscopic L5-S1 interbody fusion 1 year previously. Thinsection CT scanning revealed a nonunion (Fig. 12). Revision of the nonunion was undertaken in a minimally invasive fashion. A percutaneous approach to the L-5 transverse process and sacral ala was performed bilaterally by using the MED technique. ${ }^{3}$ Autologous bone graft

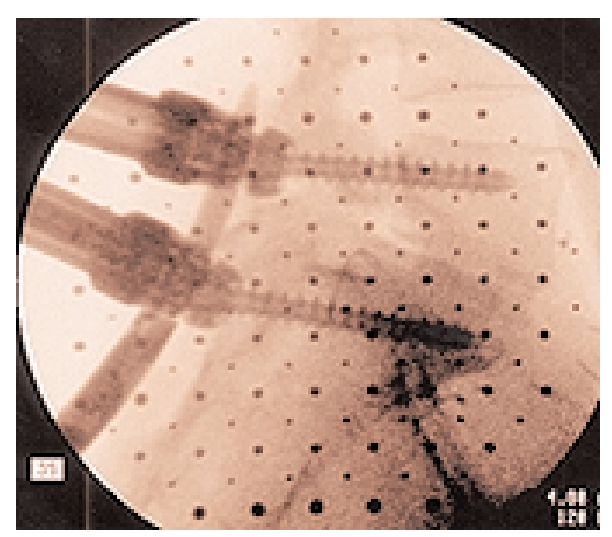

Fig. 14. An intraoperative fluoroscopic image confirming correct placement of the percutaneous rod. 


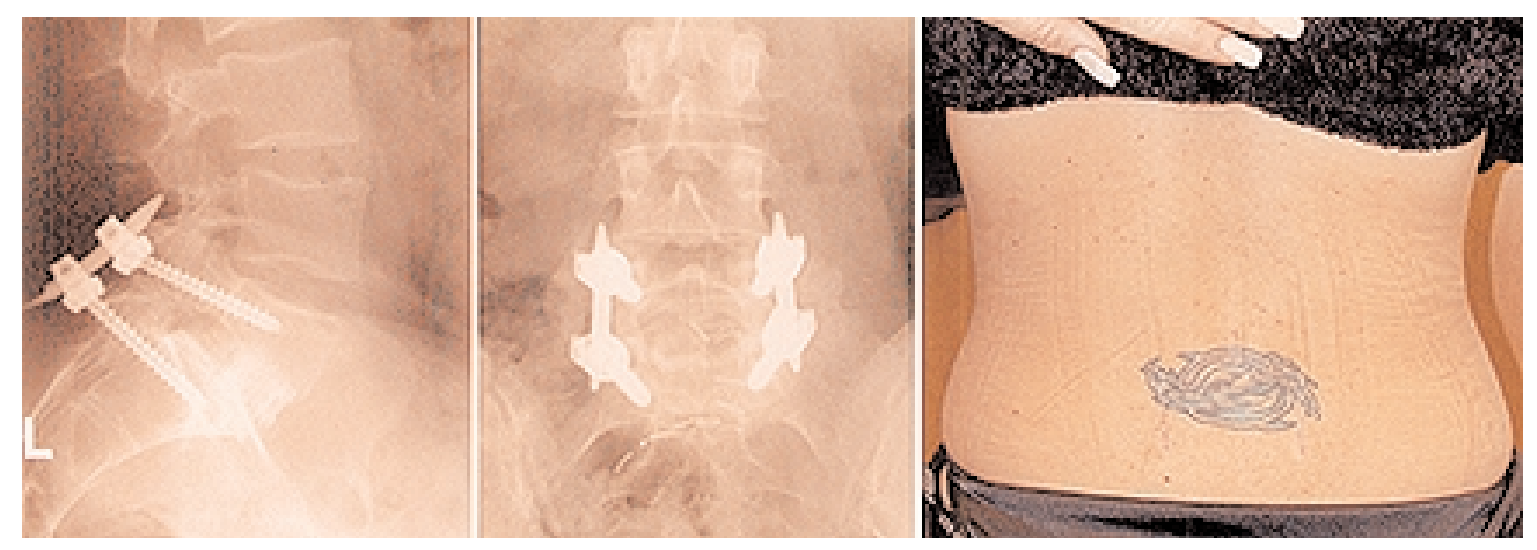

Fig. 15. Postoperative studies. Left: Lateral radiograph revealing proper positioning of the percutaneously placed screw/rod combination. Center: Anteroposterior radiograph demonstrating acceptable screw placement and bilateral posterolateral fusion. Right: Photograph showing that the small stab incisions are barely discernible. This patient's tattoo was preserved even though it was located in the lumbar midline.

was harvested by wanding the MED tube to the iliac crest. Percutaneous placement of Sextant screws was carried out through the same paramedian stab wounds that were used for the fusion. Figure 13 shows the multiplanar, virtual fluoroscopic guidance used for percutaneous placement of the left L-5 pedicle screw. A fluoroscopic image of the rod placement is shown in Fig. 14. Postoperative AP and lateral radiographs revealed good positioning of the hardware and a bilateral posterolateral fusion mass (Fig. 15 left and center). A photograph of the patient's incisions can be seen in Fig. 15 right.

\section{DISCUSSION}

Percutaneous fixation of the lumbar spine was first described by Magerl, ${ }^{6}$ who used an external fixator. Mathews and Long ${ }^{5,7}$ first described and performed a wholly percutaneous lumbar pedicle fixation technique in which they used plates as the longitudinal connectors. Lowery and Kulkarni ${ }^{5}$ subsequently described a similar technique in which rods were placed. Although the latter authors reported a high success rate, Mathews and Long noted a significant rate of nonunion (HH Mathews, personal communication, 2001). In all cases, the longitudinal connectors were placed either externally ${ }^{6}$ or superficially, just beneath the skin., ${ }^{5,7}$ This has several potential disadvantages. First, the superficial hardware can be irritating and requires routine removal. ${ }^{5}$ Second, longer screws (and thus longer moment arms) are required, producing a less effective biomechanical stabilization than that achieved using standard pedicle fixation systems and leading to a higher potential for implant failure.

The use of the Sextant system with or without virtual fluoroscopy offers several distinct advantages over conventional pedicle screw fixation. The system eliminates the need for a large midline incision and significant paraspinous muscle dissection. Both the pedicle screws and the precontoured rod are placed through stab incisions. The paraspinous muscles are bluntly split rather than divided, leading to potentially shorter periods of hospitalization and recovery. ${ }^{3}$ Blood loss and tissue trauma are minimized. An ideal lateral-to-medial screw trajectory is much more easily accomplished, especially in larger patients, as significant paraspinous tissue retraction is avoided.

Compared with previously used percutaneous techniques, the Sextant procedure allows the screw/rod to be placed in a standard anatomical position. This optimizes the biomechanics of the fixation and allows the hardware to remain in place without irritating the superficial tissues of the low back, and thus avoids routine hardware removal. In addition, this technique minimizes much of the "fiddle factor" of connecting a percutaneous rod or plate to pedicle screws. The inserter geometrically constrains the rod's pathway, thus simplifying insertion of the rod. The cannulated extension sleeves allow the lock plugs to be quickly and easily seated against the rod, simplifying screw-rod connection. Because the Sextant inserter remains connected to the screws and rods, appropriate forces (compression and distraction) can be applied to the construct prior to final tightening.

Minimally invasive approaches for performing lumbar fusion are in their infancy. Laparoscopic anterior lumbar interbody fusion has only been recently described. ${ }^{14}$ Other minimally invasive approaches to lumbar fusion, such as the posterolateral MED onlay technique described in this paper, are evolving. The goal of these surgeries, as for all minimally invasive procedures, is to minimize approachrelated morbidity while achieving the same result as more traditional, invasive approaches. The Sextant technique follows these same principles, allowing the surgeon to perform biomechanically sound internal spinal fixation with minimal tissue trauma. Coupled with techniques for minimally invasive spinal fusion and decompression, ${ }^{3}$ its clinical utility should widen. Certainly, the preliminary clinical results presented in this paper are promising.

\section{References}

1. Foley KT, Simon DA, Rampersaud YR: Virtual fluoroscopy. Op Tech Orthop 10:77-81, 2000

2. Foley KT, Simon DA, Rampersaud YR: Virtual fluoroscopy: computer-assisted fluoroscopic navigation. Spine 26:347-351, 2001 


\section{K. T. Foley and S. K. Gupta}

3. Foley KT, Smith MM: Microendoscopic discectomy. Tech Neurosurg 3:301-307, 1997

4. Gaines RW: The use of pedicle-screw internal fixation for the operative treatment of spinal disorders. J Bone Joint Surg Am 82-A:1458-1476, 2000

5. Lowery GL, Kulkarni SS: Posterior percutaneous spine instrumentation. Eur Spine J 9 (Suppl 1):S126-S130, 2000

6. Magerl F: External skeletal fixation of the lower thoracic and the lumbar spine, in Uhthoff HK, Stahl E (eds): Current Concepts of External Fixation of Fractures. New York: SpringerVerlag, 1982, pp 353-366

7. Mathews HH, Long BH: Endoscopy assisted percutaneous anterior interbody fusion with subcutaneous suprafascial internal fixation: evolution of technique and surgical considerations. Orthop Int Ed 3:496-500, 1995

8. Rampersaud YR, Foley KT, Shen AC, et al: Radiation exposure to the spine surgeon during fluoroscopically assisted pedicle screw insertion. Spine 25:2637-2645, 2000

9. Schlenzka D, Laine T, Lund T: Computer-assisted spine surgery. Eur Spine J 9 (Suppl 1):S57-S64, 2000

10. Slomczykowski M, Roberto M, Schneeberger P, et al: Radiation dose for pedicle screw insertion. Fluoroscopic versus computer-assisted surgery. Spine 24:975-983, 1999
11. Thomsen K, Christensen FB, Eiskjaer SP, et al: 1997 Volvo Award winner in clinical studies. The effect of pedicle screw instrumentation on functional outcome and fusion rates in posterolateral lumbar spinal fusion: a prospective, randomized clinical study. Spine 22:2813-2822, 1997

12. Wiesner L, Kothe R, Ruther W: Anatomic evaluation of two different techniques for the percutaneous insertion of pedicle screws in the lumbar spine. Spine 24:1599-1603, 1999

13. Yuan HA, Garfin SR, Dickman CA, et al: A historical cohort study of pedicle screw fixation in thoracic, lumbar, and sacral spine fusions. Spine 19 (Suppl 20):2279S-2296S, 1994

14. Zucherman JF, Zdeblick TA, Bailey SA, et al: Instrumented laparoscopic spinal fusion. Preliminary results. Spine 20: 2029-2035, 1995

Manuscript received February 27, 2001.

Accepted in final form March 21, 2001.

Financial support for the research in this study was provided by Medtronic Sofamor Danek.

Address reprint requests to: Kevin T. Foley, M.D., ImageGuided Surgery Research Center, 220 South Claybrook, Suite 700, Memphis, Tennessee 38104. email: kfoley@usit.net. 
Title 